\title{
Solar wind control of auroral substorm onset locations observed with the IMAGE-FUV imagers
}

\author{
J.-C. Gérard, B. Hubert, A. Grard, and M. Meurant \\ Laboratoire de Physique Atmosphérique et Planétaire, Université de Liège, Liège, Belgium
}

S. B. Mende

Space Science Laboratory, University of California, Berkeley, California, USA

Received 10 July 2003; revised 19 November 2003; accepted 16 December 2003; published 13 March 2004.

[1] The FUV imagers on board the IMAGE satellite provide multispectral snapshots of the polar region every $2 \mathrm{~min}$. The combination of the Wide-Angle Imaging Camera (WIC) with SI12 (Doppler shifted Lyman- $\alpha$ ) and SI13 (135.6 $\mathrm{nm}$ ) spectral imagers is used to discriminate between the electron and the proton aurora. We describe a statistical study of the location of 78 substorms observed close to the 2000-2001 winter solstice. The latitudinal distribution of the onsets observed with WIC is asymmetric with a median at $65.6^{\circ}$ MLAT and a full width at half maximum (FWHM) of $3.5^{\circ}$. Their local time distribution is concentrated between 2000 and 0200 MLT with a median at $23.4 \pm 0.3$ hours MLT and a FWHM of 1.8 hours. No statistically significant difference is found in the spatial distribution of the proton and electron onsets. All onsets take place within a region of preexisting proton precipitation, indicating that substorm initiation occurs in regions of stretched but dipole-like field lines that cross the equatorial plane close to the Earth. Latitudes of substorm onsets are located at a variable distance from the poleward FUV auroral boundary but remain at a nearly constant distance from the equatorward limit of both proton and electron auroral ovals. The magnetic latitudes of the onsets are correlated with some of the solar wind plasma properties measured by the ACE satellite prior to the substorm breakup. In particular, a clear anticorrelation is found between the onset MLAT and the 1-hour averaged solar wind dynamic pressure. A decrease of the onset latitude is also observed for larger B intensity values. No dependence of the onset MLT on the solar wind speed is observed, in contrast to the relationship expected from the thermal catastrophe model for substorm initiation. Our results are in agreement with models locating the initial instability in the near magnetosphere such as the near-Earth current disruption models. INDEX TERMS: 2788 Magnetospheric Physics: Storms and substorms; 2716 Magnetospheric Physics: Energetic particles, precipitating; 2704 Magnetospheric Physics: Auroral phenomena (2407); 2784 Magnetospheric Physics: Solar wind/magnetosphere interactions; 2744 Magnetospheric Physics: Magnetotail; KEYWORDS: auroral substorms, substorm onset, proton aurora, auroral boundaries, latitudinal distribution, solar wind control

Citation: Gérard, J.-C., B. Hubert, A. Grard, M. Meurant, and S. B. Mende (2004), Solar wind control of auroral substorm onset locations observed with the IMAGE-FUV imagers, J. Geophys. Res., 109, A03208, doi:10.1029/2003JA010129.

\section{Introduction}

[2] The framework for description of auroral activity was first set by Akasofu [1964], who described the auroral substorm as the basic scenario providing a global view of auroral dynamics. Since then, a considerable amount of effort has been devoted to the understanding of the physical processes occurring in the solar wind and the magnetosphere which control auroral substorm morphology, dynamical evolution and intensity. Phase 1, the growth phase, is characterized by an equatorward motion of the quiet auroral arcs near local midnight and an extension of the size of the

Copyright 2004 by the American Geophysical Union. 0148-0227/04/2003JA010129\$09.00 polar cap. Phase 2, the auroral onset (or breakup), is a sudden brightening of arcs in the late evening sector, generally near the equatorward boundary of the discrete arc system. It signals the impulsive release of energy previously stored in the magnetosphere. It is followed by an explosive expansion where the brightened arc evolves into a large-scale disturbance (the bulge) accompanied by a poleward expansion of the region of auroral emission. Finally, the third phase (recovery) corresponds to the fading of the intensity and slow return to presubstorm conditions. The substorm usually follows a southward turn of the interplanetary magnetic field (IMF). The idea that field line reconnection is the mechanism through which energy from the solar wind is transferred to the magnetosphere and subsequently dissipated in the ionosphere is 
widely accepted. In spite of several decades of continuous research and impressive development of space instrumentation and numerical modeling, the question of what process triggers a substorm onset and where it is located in the magnetosphere is still far from totally understood. The question was raised whether the optical onset detected from the ground or with satellite-borne imagers is a good detector of the onset in comparison with other indicators such as the AE magnetic index or the Pi2 pulsations. Recent studies [Liou et al., 1999] have shown that the detection of substorm onsets by global imagers is a better indicator of the initial phase leading to the development of a substorm than other ground-based proxies.

[3] Many theories and models have been proposed to attempt to account for the wealth of observational constraints provided by geosynchronous satellites, groundbased and space-borne auroral imagers. At this point, two categories of models are still debated [Lui, 2001]. In the near-Earth neutral line (NENL) model, a neutral line (X-line) is formed in the plasma sheet in the East-West direction at distances of $\sim 15-25 \mathrm{Re}$. This process causes a plasma flow towards the Earth and generates a plasmoid that is expelled away from Earth in the magnetotail. The cross-tail magnetospheric current flowing from the dawnside to the duskside of the nightside magnetosphere is diverted into the ionosphere. This current wedge causes an increase of the magnetic field normal to the equatorial plane and dipolarization of the magnetic field lines previously stretched outward. Evidence has however accumulated that the onset mechanism is located closer to Earth, at distances of 5-10 Re. Several authors [Shiokawa et al., 1997; Baker et al., 2002] proposed a modified version of the NENL paradigm where the reconnection occurs at $\sim 20 \mathrm{Re}$ but where the high-speed earthward plasma flow brakes down when it collides with the high-pressure plasma at 5-10 Re. The auroral substorm onset is observed as the consequence of this interaction. In the other view, the triggering mechanism is located in the near-Earth region $(<10 \mathrm{Re})$ and the substorm is a consequence of the current disruption that takes place in the same region. This picture is consistent with many measurements showing that magnetic field dipolarization takes place at geosynchronous distance in parallel with simultaneous substorm onset [McPherron et al., 1973].

[4] Several mechanisms have been proposed to explain the instability triggering the onset: ballooning instability, Kelvin-Helmholtz instability, kinetic instability, magnetosphere-ionosphere coupling. One of them, the thermal catastrophe model (TCM) [Goertz and Smith, 1989], assumes that onsets are caused by a sudden impulsive increase of the plasma temperature near the plasma sheet boundary layer (PSBL) resulting from the conversion of energy of magnetoacoustic waves from the tail lobes into kinetic energy of Alfven waves. The magnetoacoustic waves in the lobe are generated by surface fluctuations on the high-latitude magnetopause resulting from KelvinHelmholtz waves excited by the interaction of the solar wind with the magnetosphere.

[5] Henderson and Murphree [1995] studied the location of the regions where onset instabilities can occur in the framework of the TCM. They found, using a realistic tail field model, that (1) the onset region extends into the deep tail only for high solar wind speeds and (2) the onset local time shifts toward midnight for increasing solar wind speeds. In a recent study based on IMAGE-FUV data and parallel FAST in situ measurements, Mende et al. [2003] found that electrons precipitating in the leading edge of the auroral surge showed characteristics of acceleration by Alfven waves, presumably resulting from field line dipolarization. In contrast, equatorward of the surge, quasi-static "inverted V" structures were imbedded into a broader region of electron precipitation. The FAST measurements showed that the onset location was separated from the closed-open field line boundary by an extended region of closed field lines with plasma sheet precipitation.

[6] In this work, we investigate the latitudinal and MLT distributions of breakup initiation. The magnetic latitude of onsets is the footprint of the magnetospheric region where the sudden release of energy leads to precipitation of auroral particles. The relative position of the substorm onset and proton precipitation yields important information concerning where the onset field lines map in the equatorial plane. Murphree et al. [1993] mapped the latitude of onsets measured with Viking using the Tsyganenko [1987] geomagnetic field model. They found that the onsets occur far equatorward of the open-closed field line boundary and that the higher latitude emission is undisturbed until it is reached by the poleward expansive bulge of the substorm. Lyons et al. [2002] found that auroral onsets generally occur along a thin breakup arc forming equatorward of all growth phase arcs a few minutes prior to onset. These results imply that the processes responsible for auroral breakup initiate a few minutes prior to the time normally identified as substorm expansion-phase onset. They also indicate that the process that triggers the onset of substorms does not require the occurrence of plasma sheet changes, significant enough to affect magnetosphere-ionosphere electrodynamics, along field lines that cross the equator tailward of the substorm onset region. Frank and Sigwarth [2000] analyzed the location of six onsets observed in visible light with the Visible Camera on board POLAR. They found that the initial events occurred near 9-10 $R_{E}$ for low Kp conditions and between 4.7 and $6.6 \mathrm{R}_{\mathrm{E}}$ for more active periods. These results suggest that onsets occur at shorter distances from Earth in more disturbed conditions, possibly as a consequence of solar wind control of the region of the initial instability. Recently, Dubyagin et al. [2003] presented evidence based on FAST satellite measurements crossing substorm onset combined with ground-based observations. They found that the onset was launched in the near-Earth domain near $8 \mathrm{R}_{\mathrm{E}}$, a location that is not consistent with magnetic reconnection as the direct cause of the breakup.

[7] A substorm onset described by Frank and Sigwarth [2000] was found to occur in a region of hydrogen emission, indicating that the field line threading the onset spot was closed and connects to the ring current region. Similar ground-based observations with meridian scanning photometers lead to the conclusion that the brightening of the equatorward arc at onset is imbedded into a region of diffuse $\mathrm{H}_{\mathrm{B}}$ proton induced emission [Samson et al., 1992]. Consequently, onsets occur on stretched but dipole-like field lines connecting to a region of energized proton population. The equatorward boundary of the proton aurora corresponds to the limit between isotropic (more tailward) and aniso- 
tropic (more Earthward) energetic protons in the magnetotail [Donovan et al., 2003]. The isotropic boundary (IB) marks the separation between regions of adiabatic and nonadiabatic proton motion in the neutral sheet. Equatorward of the IB, the proton pitch angle distribution measured near $1000 \mathrm{~km}$ is isotropic outside of the mostly empty upgoing and downgoing loss cones and ions are trapped with little or no pitch angle scattering. Poleward of the IB, strong pitch angle diffusion occurs due to the curvature of the field lines relative to the proton gyroradius and produces diffuse proton auroral emission of hydrogen lines.

[8] The study of correlations of onset characteristics with solar wind parameters can provide useful constraints on the conditions leading to the initiation of substorms in the magnetotail. A study by Liou et al. [2001] based on 10 months of POLAR UVI observations has shown that seasonal effects are observed on the distribution of the onset locations. In particular, the substorm latitudinal distribution is more symmetric in winter than in summer and onsets tend to start at earlier local times in summer than in winter. Correlations of onset positions with the IMF components averaged over the 1-hour period prior to onset have shown that the sign and value of $\mathrm{B}_{\mathrm{z}}$ controls the onset magnetic latitude, whereas the onset local time mostly depends on the $\mathrm{B}_{\mathrm{y}}$ component, with a weaker dependence on $\mathrm{B}_{\mathrm{x}}$.

[9] In this study, we determine the latitude and local time distributions of electron and proton onsets. We investigate the location of the onsets relative to the boundaries of the FUV oval prior to the onset. We examine the dependence of the position of the onsets on the solar wind and IMF parameters and discuss implications in terms of source regions in the magnetotail.

\section{Auroral Onset Identification and Location}

[10] The Imager for Magnetopause-to-Aurora Global Exploration (IMAGE) satellite was launched in March 2000 to investigate the response of the magnetosphere to the time variable solar wind [Burch et al., 2001]. It is in a highly eccentric orbit with an initial perigee altitude of $\sim 1000 \mathrm{~km}$ and an apogee of about 7 Earth radii. The FUV instrument includes three imagers observing the global north aurora [Mende et al., 2000]. The Wideband Imaging Camera (WIC) imager has a passband between 140 and $180 \mathrm{~nm}$. It is mostly sensitive to the LBH bands and the NI $149.3 \mathrm{~nm}$ line but also includes a small contribution from the OI $135.6 \mathrm{~nm}$ and NI $174.3 \mathrm{~nm}$ lines. Excitation of the LBH bands and NI lines is produced by collisions between primary electrons, protons, and secondary electrons with $\mathrm{N}_{2}$ molecules. The WIC CCD camera outputs the information digitally in the form of 16-bit $\mathrm{AD}$ units. The Spectrographic Imager (SI) is a two-channel narrow-band imager of the auroral emissions at $121.8 \mathrm{~nm}$ and $135.6 \mathrm{~nm}$. From apogee, each WIC pixel intercepts a $40 \times 40 \mathrm{~km}^{2}$ area projected on the Earth. SI12 images the brightness of Doppler shifted Lyman- $\alpha$ (Ly- $\alpha$ ) auroral emissions. As precipitating protons collide with neutral atmospheric constituents they can capture an electron and become fast hydrogen atoms. A fraction of the fast $\mathrm{H}$ atoms are produced in the $\mathrm{H}(2 \mathrm{p})$ state and radiate Doppler-shifted Ly- $\alpha$ photons. The SI12 imager efficiently rejects the geocoronal $\mathrm{Ly}-\alpha$ emission at $121.56 \mathrm{~nm}$ that would otherwise dominate the signal and only responds to proton precipitation [Mende et al., 2000; Gérard et al., 2001]. An important feature of IMAGE-FUV is the simultaneity of the multispectral imaging and its ability to discriminate proton and electron aurora. SI13, the second spectral imager, is centered on $135.6 \mathrm{~nm}$ with a $5 \mathrm{~nm}$ wide passband. This window includes the OI $135.6 \mathrm{~nm}$ doublet and a few adjacent LBH bands. Detailed modelling has shown that the $135.6 \mathrm{~nm}$ emission contributes $40-60 \%$ of the SI13 count rate, depending on the particle energy [Hubert et al., 2002]. From apogee, an SI pixel intercepts a $75 \times 75 \mathrm{~km}^{2}$ area. A snapshot of the global north auroral emission is obtained simultaneously by the three imagers every $121 \mathrm{~s}$. The effective exposure time is approximately $10 \mathrm{~s}$ for WIC and $5 \mathrm{~s}$ for both SI12 and SI13. Performances of the FUV imagers (field of view, point spread function, and absolute sensitivity) were determined by laboratory calibrations and in-flight observations of hot stars of known spectral brightness [Gladstone et al., 2000; Frey et al., 2003]. The width of the PSF was evaluated from the observation of hot stars crossing the field of view and was found to be about 3 WIC pixels and 3.5 SI12 pixels, corresponding to an effective linear resolution of $\sim 120 \mathrm{~km}$ and $220 \mathrm{~km}$ from apogee, respectively. The overall sensitivity of each imager is daily controlled by monitoring the instrumental response to reference stars routinely visible in the imagers' field of view.

[11] All FUV data available from 19 November 2000 to 15 January 2001 have been visually examined to identify nightside auroral onsets. For this purpose, all WIC raw data collected during this winter solstice period are looked at using the FUV database and visualization software. For each substorm, we verify that the event develops into a full poleward and longitudinal expansion, so that short-lived pseudo-breakups are excluded. The onset time is obtained by going back in time to the initial, fairly localized initial brightening. The magnetic latitude (MLAT) and local time (MLT) coordinates of the brightest pixel, usually at the center of the brightening spot, are saved in a database for statistical analysis. Intensifications occurring within $30 \mathrm{~min}$ following a previous breakup are not considered to avoid mixing new substorms and local reactivation of existing auroral emission from a previous evolving substorm. The uncertainty on the time of the actual onset is $2 \mathrm{~min}$ from the observed time of initial brightening. It should be noted that at the spatial and temporal resolution of global FUV imagers, the onset is detected by brightening of the substorm vortex following the initial intensification of the equatorward arc that is generally below the detection threshold of global FUV imagers. The delay of a few minutes that may occur between the two events therefore limits the detection of the true moment of the breakup [Voronkov et al., 2003].

[12] Once the characteristics of the WIC observation of an onset are determined, the same procedure is applied to the SI13 and SI12 observations. All WIC and SI13 intensifications are colocated within the observational uncertainties, as expected for emissions both excited by electron precipitation. The SI12 onsets are unambiguous signatures of the initialization of proton precipitation since Doppler shifted Ly- $\alpha$ emission is not excited by auroral electrons. The universal time, geographic latitude and longitude, MLAT, and MLT coordinates of the proton onsets are 

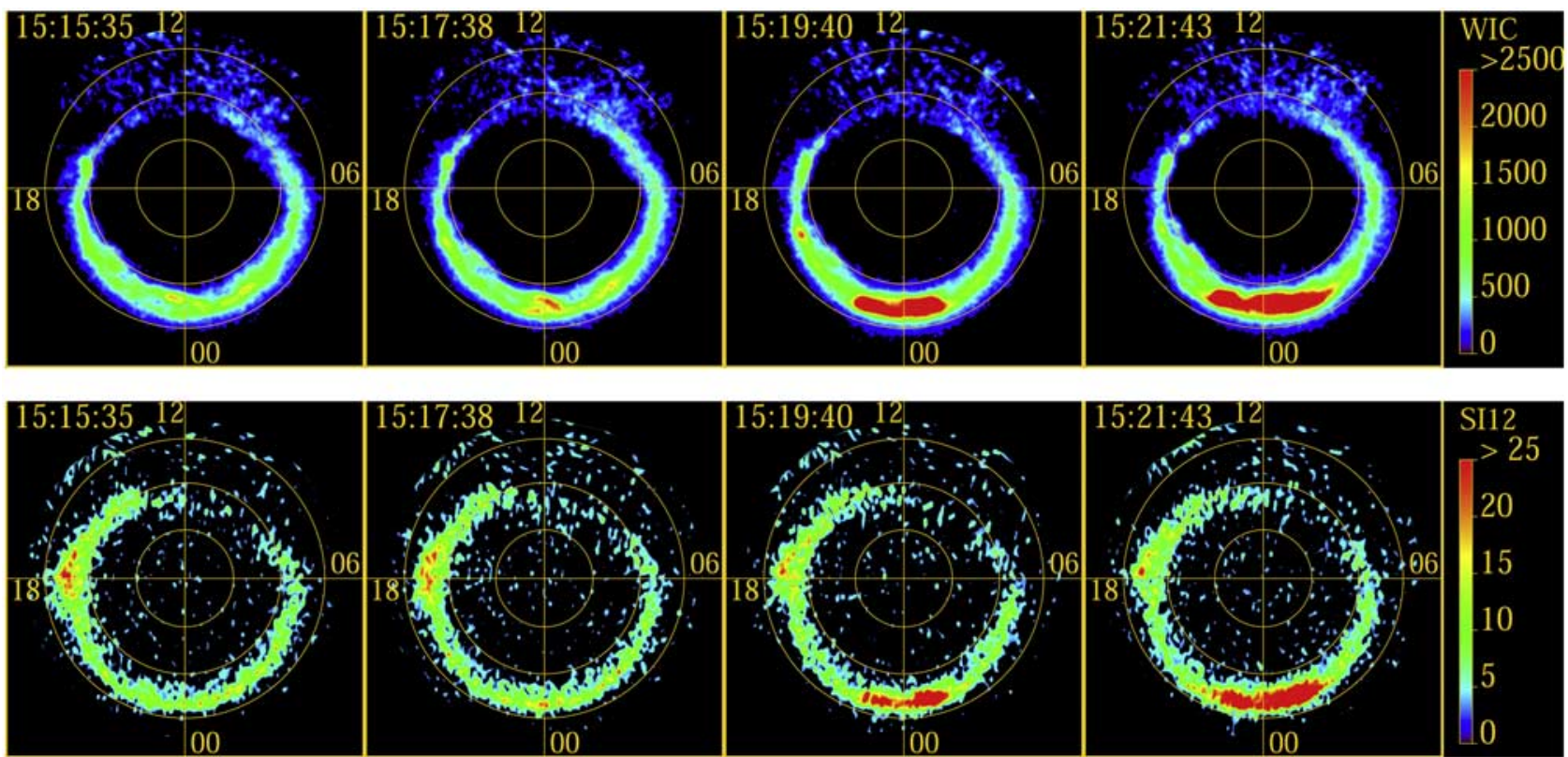

Figure 1. Sequence of four consecutive FUV images of a substorm onset observed on 6 December 2000. The top panel shows remapped (MLAT, MLT) WIC images. The magnetic parallels are spaced by $10^{\circ}$ MLAT and the meridians by 6 hours of magnetic longitude. The first image shows the pre-onset auroral oval. At 1517:38 UT, a substorm onset is clearly identified near magnetic midnight. It intensifies and expands as seen on the last two frames. The bottom panel shows the SI12 observations. It should be noted that the initial onset brightening is located within a region of preexisting proton precipitation.

provided to the onset database. The magnetic coordinate system used to order FUV observations is the Apex coordinate system described by Richmond [1995]. The accuracy associated with the selection of the onset location in the image is on the order of 2 WIC pixels, corresponding to $\sim 1.5^{\circ}$ of latitude at $60^{\circ}$ for observations near apogee.

[13] A total of 84 onsets were observed with WIC and SI13 during the 4-week period analyzed in this study. A subset of 78 cases when onsets were in the fields of view of both WIC and SI12 will be considered. An example of substorm breakup observed with WIC and SI12 is shown in Figure 1. The polar projections of the north polar region show a sequence of four consecutive WIC and SI12 images. At 1515:35 UT, the auroral oval is weakly visible in both wavelengths as it corresponds to the end of a period of low solar wind activity and northward IMF. Two minutes later, the start of the explosive expansion of the aurora is visible as an intensification in WIC near magnetic midnight at $67^{\circ}$ MLAT. At this time, the SI12 imager observes a weak intensification of auroral Ly- $\alpha$. The next frames show the initiation of both the electron and proton substorms. Finally, the last WIC and SI12 frames show a continuing intensification and poleward expansion associated with the continuing auroral substorm development.

\subsection{Latitudinal and Local Time Distribution of Onsets}

[14] We first examine the distribution of onsets observed by WIC (electron precipitation) and compare it with earlier studies, both to validate our procedure and to specify some of the features specific to this dataset.

\subsubsection{WIC Data}

[15] The distribution of electron auroral onset in magnetic latitude is shown in Figure 2 as a histogram with
MLAT bins one degree wide. The distribution shows a peak at $66.5^{\circ} \pm 0.5^{\circ}$. The mean is $65.4^{\circ} \pm 0.6^{\circ}$ and the median of the distribution is $65.6^{\circ}$ with a full width at half maximum (FWHM) of $3.5^{\circ}$. The distribution is clearly asymmetric with a longer tail toward the low latitudes than poleward. No onset was observed below $58^{\circ}$ or above $71^{\circ} \mathrm{N}$. The skewness of the MLAT distribution of onsets was previously observed by Liou et al. [2001]. It probably

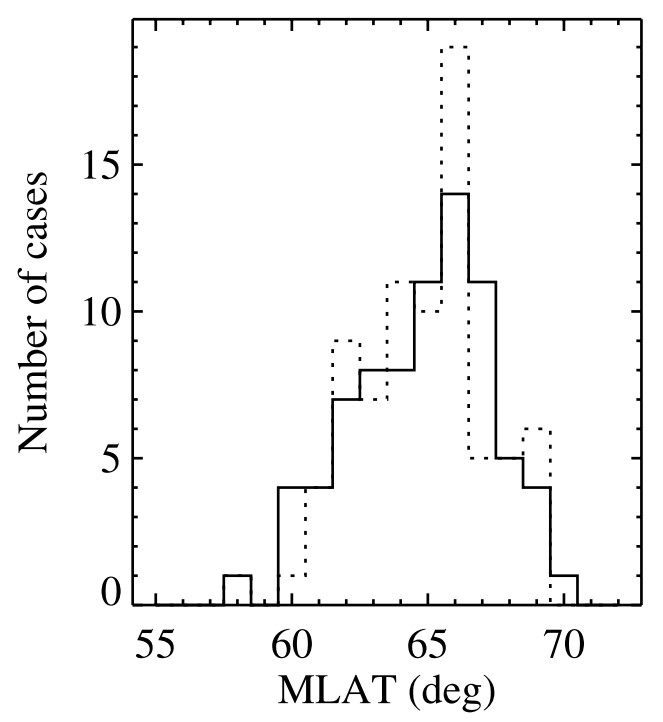

Figure 2. Distribution of occurrence of onsets with respect to magnetic latitudes (MLAT) observed by WIC for electron precipitation (solid line) and by SI12 for proton precipitation (dashed line) near winter solstice. 


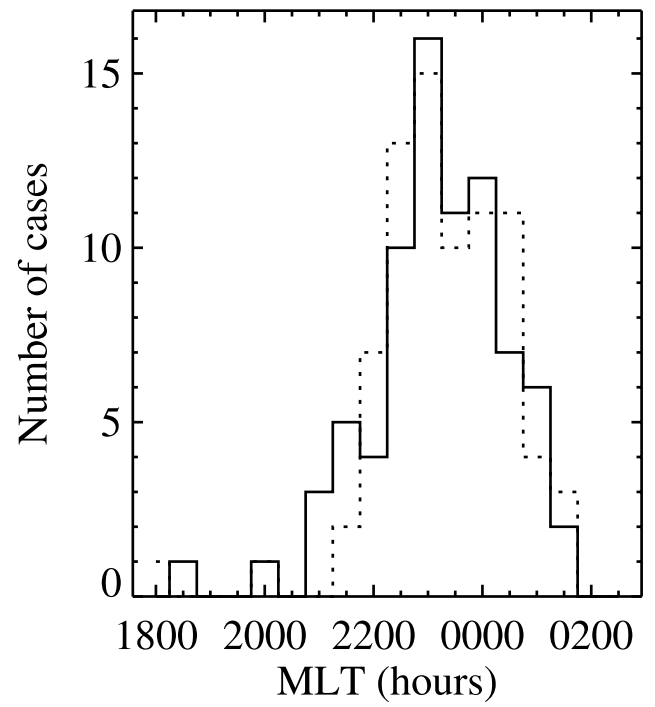

Figure 3. Distribution of occurrence of onsets with respect to magnetic local time (MLT) observed by WIC for electron precipitation (solid line) and by SI12 for proton precipitation (dashed line) near winter solstice.

reflects the nonlinear dependence of the MLAT of the magnetic field line footprint with respect to the distance in the equatorial plane.

[16] Figure 3 shows the magnetic local time distribution of substorm breakups displayed with 0.5 -hour bins. It is more symmetric than for MLAT, with a mean and a median of $23.4 \pm 0.3$ hour MLT. The FWHM of the distribution $\sim 1.8$ MLT hour. Of the 78 cases, 51 occurred before midnight, indicating a clear bias toward premidnight hours. Except for one case, no onset was observed before 2000 or later than 0200 MLT. The dependence of the onset local time with the IMF $\mathrm{B}_{\mathrm{y}}$ component will be examined in section 3.2.

\subsubsection{SI12 Data}

[17] The MLAT distribution of proton auroral onsets is shown for comparison in Figure 2. The distribution appears quite similar to that of the electron onsets. The mean and median values are $64.8^{\circ} \pm 0.5^{\circ}$ and $65.0^{\circ}$, respectively. The FWHM of the MLAT of proton onset distribution is $3^{\circ}$, quite close to the electron distribution. The width of the electron and proton MLAT distribution are thus identical, within the statistical uncertainty. The $0.6^{\circ}$ difference between the peaks of the two distributions is not statistically significant at the $90 \%$ confidence level.

[18] The results for the MLT distribution of proton onsets are plotted in Figure 3. Comparison with the electron onsets shows a somewhat different shape with a steeper drop of occurrences before 2230 MLT for protons than electrons. However the central values $($ mean $=23.55 \pm 0.3$ MLT, median $=2350 \mathrm{MLT}$ ) are within $9 \mathrm{~min}$ from the values for the electron onsets. This MLT difference corresponds to 3 degrees of longitude, which translates into $180 \mathrm{~km}$ or $2-$ 3 WIC pixels for a feature at $65^{\circ} \mathrm{N}$ observed near apogee during this period of the IMAGE mission.

[19] From a statistical standpoint, the question whether the electron and proton onset distributions are different may be addressed using a Kolmogorov-Smirnov test (K-S test). When applied to the two MLAT datasets, the K-S test leads to the conclusion that the null hypothesis (the two distributions are identical) has a probability of $51 \%$ and cannot be rejected. On this basis, it can be concluded that the proton and electron onset MLAT distributions are statistically identical. For the MLT values, the K-S test yields a p-value of 0.52 , again indicating that the proton and electron distributions may be identical. From these results, we conclude that the MLAT distributions of auroral onsets are not significantly different for electrons and protons. However, these results do not exclude the possibility that smaller scale $(<100-200 \mathrm{~km})$ differences exist between the locations of initial brightening of the two types of auroral emissions. This point will be examined in the discussion section. We now examine the relative timing of the appearance of electron and proton onsets viewed by the WIC and SI12 images.

\subsection{Onset Positions Within the Auroral Oval}

[20] The location of the substorm onsets with respect to the poleward and equatorward boundaries of the oval during the period immediately preceding breakup initiation provides some clue on the source region in the magnetotail. In particular, the question whether the onsets occur at fixed distances from the poleward boundary of the auroral emission is examined. It is widely accepted that the poleward auroral emission (PAE) boundary is closely connected to the b5e boundary of the DMSP classification, which corresponds to the transition from closed to open magnetic field lines. Similarly, the equatorward auroral emission (EAE) limit is generally coincident with the b2e boundary of the DMSP automated classification [Kauristie et al., 1999] and is often associated with the earthward edge of the main plasma sheet [Galperin and Feldstein, 1991].

[21] To examine this question, we first need to define these boundaries on the basis of the latitudinal distribution of the FUV auroral emissions (for electrons and protons). Two approaches to determine these boundaries have been used in the literature. The first one uses a threshold method [Frank and Craven, 1988; Brittnacher et al., 1999] where a constant preset brightness level is used to define regions inside the oval. The second [Kauristie et al., 1999] uses a fixed ratio to the maximum brightness observed in a meridian section. Baker et al. [2000] discussed the results of the two methods applied to images obtained with the Ultraviolet Imager (UVI) onboard the Polar spacecraft. They compared the PAE boundaries determined with the two methods to the precipitation boundary automatically derived from Defense Meteorological Satellite Program (DMSP) spectrograms [Newell et al., 1996]. They found that the PAE boundaries determined with the ratio method presented a slightly better correlation with the DMSP b5e (poleward boundary of the discrete auroral oval) than the threshold method. For this study, we first average the three WIC and SI12 images immediately preceding each auroral onset. A meridian cut through the oval is extracted at the MLT where the auroral onset will occur soon later. The poleward and equatorward oval electron (proton) boundaries are determined as the latitudes where the WIC (SI12) count rate drops below $10 \%$ of the brightness maximum on both sides of the latitudinal distribution. Visual inspection confirmed that all boundaries determined this way correspond to the poleward and equatorward limits of the FUV oval. For comparison, the threshold method was also applied using threshold values of 
50 counts/pixel $(0.5 \mathrm{kR}$ of LBH emission) for WIC and 2 counts/pixel ( $0.15 \mathrm{kR}$ of Ly- $\alpha$ emission) for SI12. For a nadir observation, these limits correspond to a flux of approximately $0.2 \mathrm{~mW} / \mathrm{m}^{2}$ of electron precipitation and $0.04 \mathrm{~mW} / \mathrm{m}^{2}$ for protons respectively.

[22] The results for the relative location of the electron and proton onsets within the FUV ovals are illustrated in Figures 4 and 5, respectively. In these plots, the PAE and EAE limits were determined using the ratio method but nearly identical results were obtained with the threshold approach. Figure 4a examines the question whether the onsets occur at a fixed (latitudinal) distance from the poleward FUV oval boundary. The plot clearly shows that the onset latitude does not covary with the PAE boundary for electron precipitation. Instead, the latitudinal distance between the onset and the poleward oval boundary increases as the onsets move equatorward, suggesting that the breakup source region does not covary with the open-closed field line boundary. Figure $4 \mathrm{~b}$ shows the location of the electron onset with respect to the equatorial oval boundary. The position of the initial substorm breakup is clearly correlated with the location of the EAE boundary determined from the WIC images $(\mathrm{R}=0.86)$. Figure $4 \mathrm{c}$ shows the distance of the onset from the WIC EAE boundary. As expected from Figure $4 \mathrm{~b}$, this distance is not correlated with the onset latitude $(\mathrm{R}=0.21)$. Figures $5 \mathrm{a}, 5 \mathrm{~b}$, and $5 \mathrm{c}$ show similar plots for proton onset locations and PAE and EAE boundaries determined from SI12 images. As expected, the results are similar to the electron onset analysis. The distance between the breakup initiation and the poleward limit of the oval increases when onsets occur at lower latitudes. The onset latitude and the SI12 EAE boundary move in parallel $(\mathrm{R}=0.91)$, keeping a mean distance of $3^{\circ}-4^{\circ}$ from each other over an excursion of $\sim 10^{\circ}$ of the equatorward boundary. Summarizing Figures 4 and 5, the electron and proton onset locations nearly move in parallel with the equatorward boundary of the preexisting oval prior to the initiation of the breakup phase. By contrast, they do not occur at a fixed distance from the poleward boundary of the oval defined by its electron or proton emission characteristics. This result strongly suggests that the location of the region of the substorm instability is largely independent of the open-closed field line boundary in the magnetotail. Instead, it occurs in a region of the main plasma sheet that moves toward or away from the Earth in parallel with the Earthward boundary of the plasma sheet.

\subsection{Time Distribution of Onsets}

[23] A specificity of the IMAGE-FUV imaging system is the quasi simultaneity of the global snapshots obtained

Figure 4. Relationship between the observed onset latitude (Mlat $\left.{ }_{\text {onset }}\right)$ and the WIC polar $\left(\mathrm{FUV}_{\mathrm{po}}\right)$ and equatorial $\left(\mathrm{FUV}_{\mathrm{eq}}\right)$ auroral boundaries and linear regression fits (solid lines). The onset latitude is plotted versus: (a) the distance between the WIC polar emission boundary and the onset latitude $(\mathrm{R}=-0.76)$; (b) the latitude of the equatorial WIC polar emission boundary $(\mathrm{R}=0.86)$ and $(\mathrm{c})$ the distance between the WIC equatorial emission boundary and the onset latitude $(\mathrm{R}=0.21)$. The dashed line in panel (b) shows the condition $\mathrm{MLAT}_{\text {onset }}=\mathrm{FUV}_{\mathrm{eq}}$.
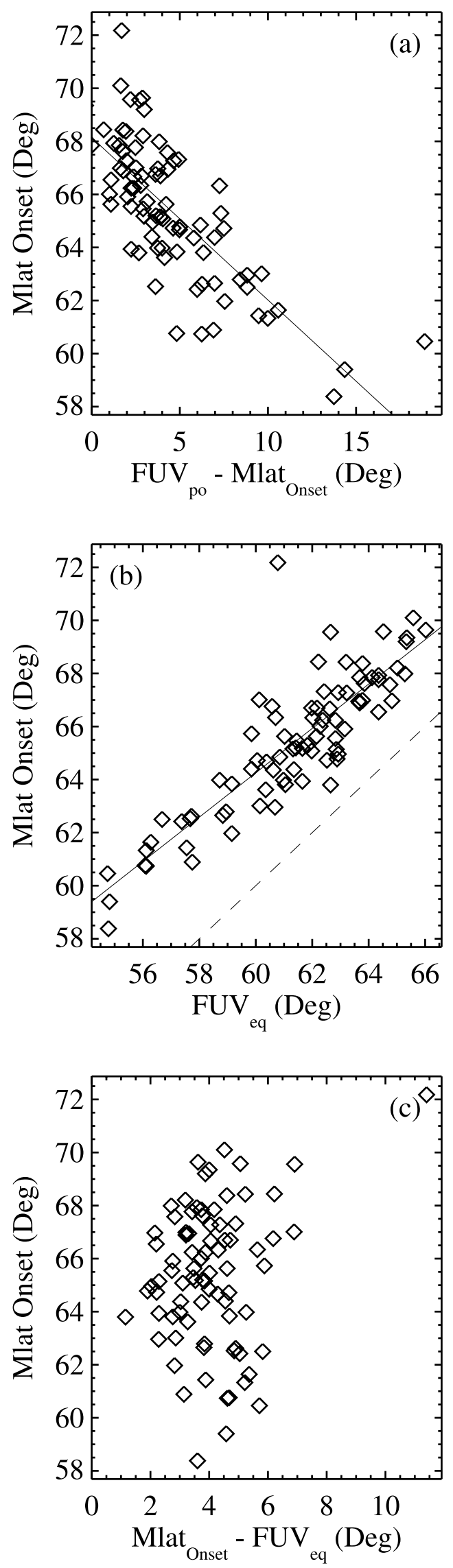

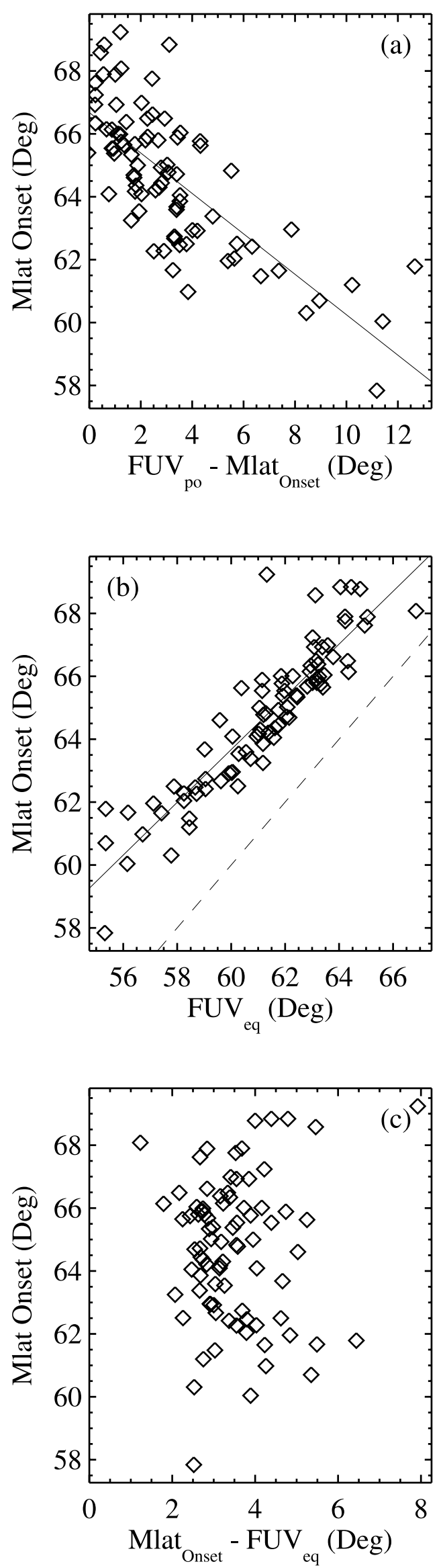

every 2 min. As mentioned before, the WIC and SI12 integration periods to construct an image are $10 \mathrm{~s}$ and $5 \mathrm{~s}$, respectively but the central times of the exposures are coincident. The time distribution of WIC and SI13 auroral onsets are found statistically identical. Among the 78 WIC and SI12 onsets, 47 occurred simultaneously, 4 protons onsets preceded the electron onsets and in 27 cases, the electron brightening is detected at least 2 min before the protons. However, the different sensitivity threshold of the two imagers makes it difficult to conclude definitely on this question since the SI12 imager does not necessary pickup the enhancement of the weak Ly- $\alpha$ emission indicating the very start of the auroral onset.

\section{Correlations With Solar Plasma and IMF Parameters}

[24] We now examine possible correlations between the solar wind plasma and the interplanetary magnetic field (IMF) parameters measured during the period preceding the onsets and the location of these initial brightening in the auroral region. For this purpose, we use data collected with the Solar Wind Electron Proton Alpha Monitor (SWEPAM) and Magnetic Field Experiment (MAG) Instruments on board the Advanced Composition Explorer (ACE) satellite. The spacecraft is located at the L1 Langrangian point approximately $1.4 \times 10^{6} \mathrm{~km}$ from Earth in the sunward direction. Past studies have shown that the time delay of onset to changes in the solar wind parameters is on the order of 20 to 60 min [Meng et al., 1973; Liou et al., 1999; Zhou and Tsurutani, 2003]. For this study, the original ACE measurements of the proton density $(\mathrm{N})$, solar wind bulk velocity (v), $B_{x}, B_{y}, B_{z}$, IMF components, and $B$ intensity are first propagated at the solar wind speed to the subsolar magnetopause. A 6-min delay is added to account for the additional time necessary to reach the auroral ionosphere. The IMF parameters are subsequently averaged over $20 \mathrm{~min}$, $40 \mathrm{~min}$, and $60 \mathrm{~min}$ periods preceding the time of the onsets observed by FUV. For most of the following study, we only use the WIC data since, as illustrated before, no significant difference was found between the WIC and SI12 MLAT spatial distribution of onsets. In addition, the study of the separation between onsets and oval limits has confirmed that both types of onsets indeed show the same behavior. Therefore the following results apply both to electron and proton breakups.

\subsection{Solar Wind Plasma}

[25] Figure 6 shows the relationship between the 1-hour averaged solar wind plasma properties $\mathrm{v}, \mathrm{N}$ and dynamic

Figure 5. Relationship between the observed onset latitude (Mlat $\left.{ }_{\text {onset }}\right)$ and the SI12 polar $\left(\mathrm{FUV}_{\mathrm{po}}\right)$ and equatorial $\left(\mathrm{FUV}_{\mathrm{eq}}\right)$ auroral boundaries and linear regression fits (solid lines). The onset latitude is plotted versus: (a) the distance between the SI12 polar emission boundary and the onset latitude ( $\mathrm{R}=-0.76)$; (b) the latitude of the equatorial SI12 polar emission boundary $(\mathrm{R}=0.91)$ and $(\mathrm{c})$ the distance between the SI12 equatorial emission boundary and the onset latitude $(\mathrm{R}=0.04)$. The dashed line shows the condition $\mathrm{MLAT}_{\text {Onset }}=\mathrm{FUV}_{\mathrm{eq}}$. 

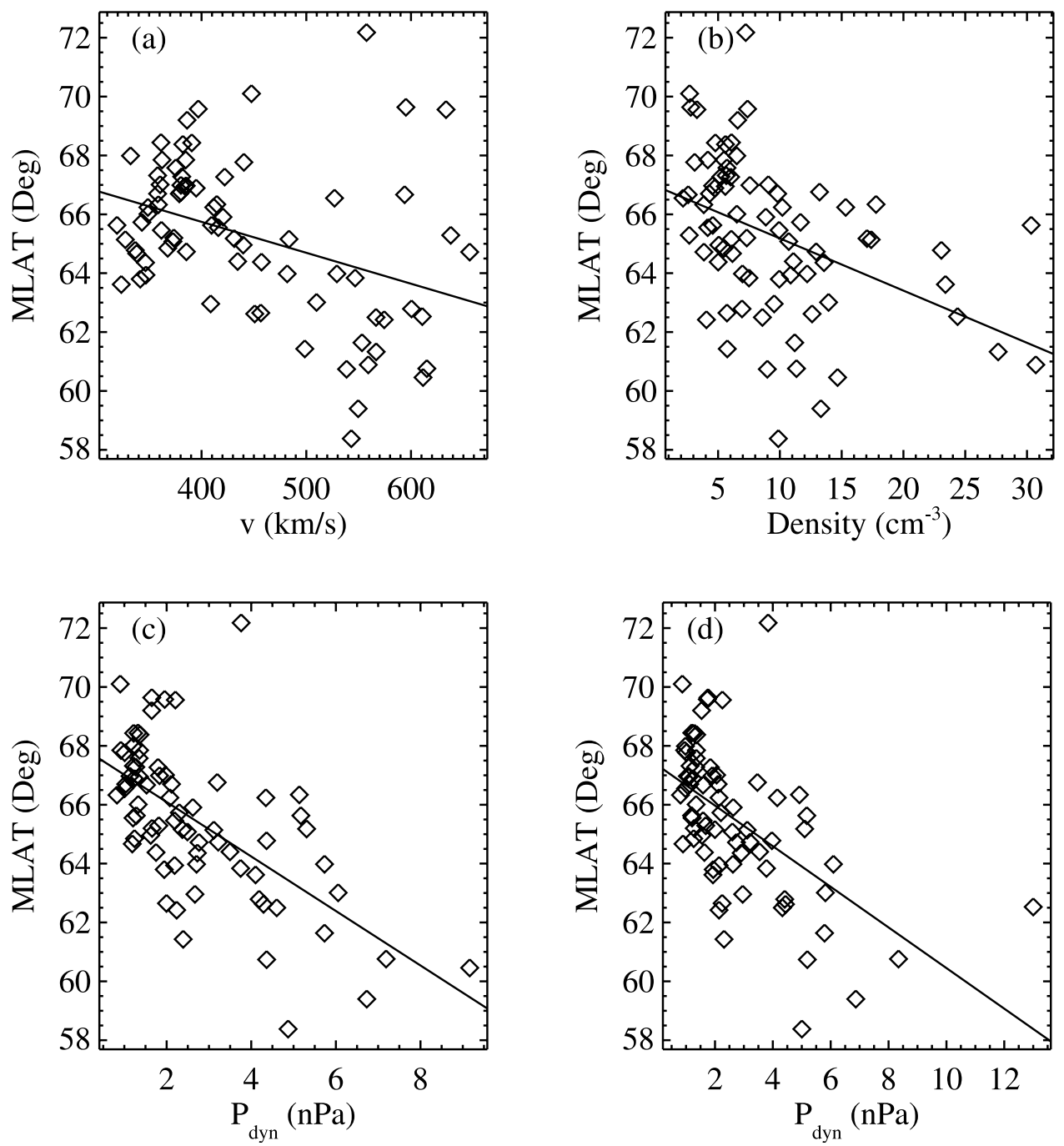

Figure 6. Correlations between the magnetic latitude of onsets and 1-hour averaged solar wind plasma parameters: (a) velocity, (b) density and (c) dynamic pressure. Figure 6d shows the correlation with the 20-min averaged value of $\mathrm{P}_{\mathrm{dyn}}$.

pressure Pdyn, and the magnetic latitude of the onset. A total of 76 cases were found when ACE data were continuously available during the hour preceding the onset observed with FUV. For this sample size, a correlation is significant at the $95 \%$ confidence level if the correlation coefficient $\mathrm{R}$ exceeds 0.22 . An anticorrelation $(\mathrm{R}=-0.38)$ is found with the solar wind speed but the correlation is higher with the density $(\mathrm{R}=-0.43)$ and Pdyn $(\mathrm{R}=-0.62)$ hourly averaged values, indicating that the compression of the magnetosphere controls the latitude of the onset. For the latter, the linear regression fit with 20-min averaged Pdyn values $(\mathrm{R}=-0.56)$ is shown in Figure $6 \mathrm{~d}$. No large difference is found between correlation coefficients for 1-hour and 20-min averaged Pdyn or between the terms of the linear regression. However, as may be expected, the range of Pdyn values is smaller for the 1-hour means. The same conclusion holds for correlations with other parameters: the correlation is quite similar when solar wind parameters are averaged over 20,40 , or $60 \mathrm{~min}$. The general conclusion drawn from these results is that more active solar wind conditions generate onsets at lower magnetic latitudes.
[26] The linear relationships between the 1-hour averaged solar wind Pdyn and MLAT is given by

$$
\operatorname{MLAT}=67.94^{\circ}-0.93^{\circ} \times \operatorname{Pdyn},
$$

where Pdyn is the hourly averaged dynamic pressure in $\mathrm{nPa}$. A similar negative correlation is found between $\mathrm{Kp}$ and the MLAT of the onset (not shown). However, we believe solar wind plasma parameters are more suitable indicators of the source processes controlling the location of onsets that the 3 -hour average $\mathrm{Kp}$ index. The $\mathrm{Kp}$ value for a given onset may include activity occurring before, during or after the substorm.

[27] The relationship between the open-closed field line boundary and the onset latitude in section 2.2 on one hand and the Pdyn-onset latitude correlation on the other hand indicates that as the solar wind dynamic pressure contracts the magnetosphere the onset moves away from the limit between open and closed field lines in the magnetotail. This point is further investigated and illustrated by Figure 7 showing the variation of the onset distance from the WIC 

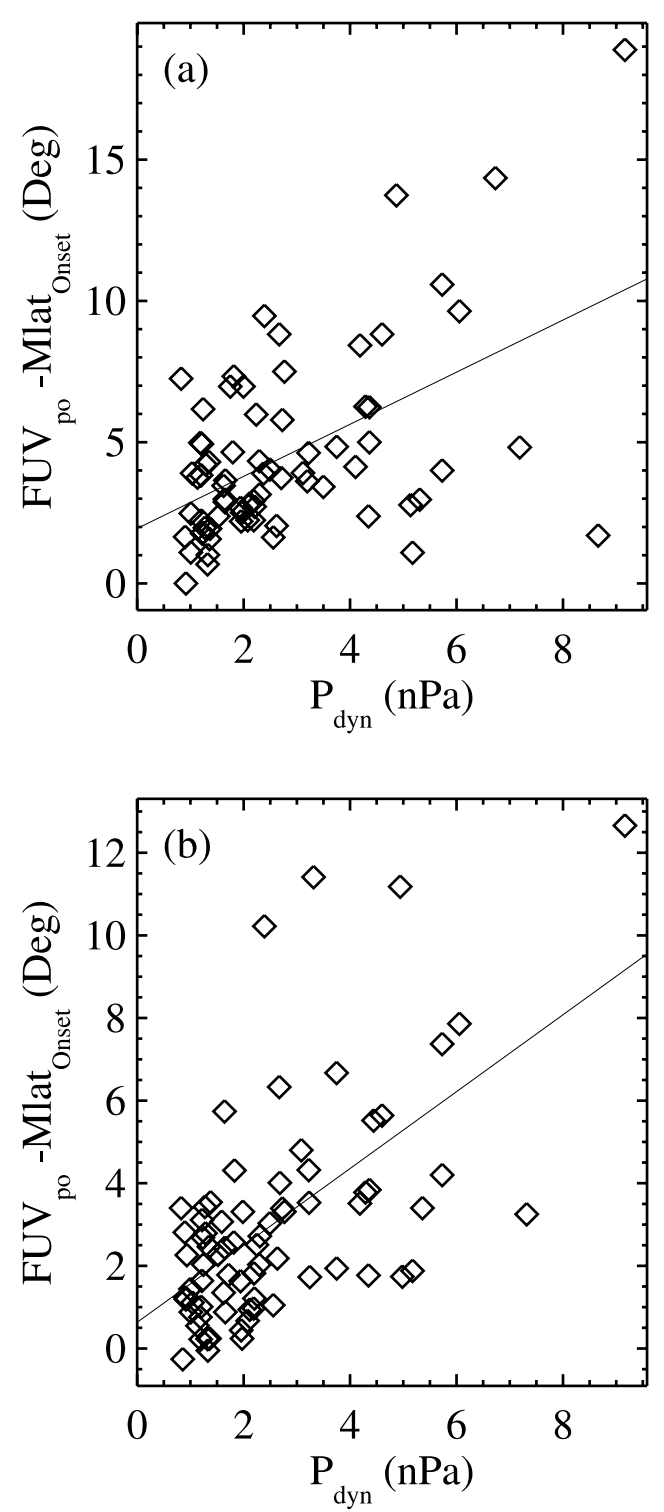

Figure 7. Variation of the distance between the WIC (a) and SI12 (b) polar emission boundary and the onset latitude with the 1-hour averaged dynamic pression before the substorm initiation.

and SI12 poleward boundaries. It is found that the distance is significantly correlated with the 1-hour averaged dynamic pressure both for electron $(\mathrm{R}=0.41)$ and protons $(\mathrm{R}=0.59)$. This result implies that when the solar wind contracts the magnetosphere, the onset region maps further away from the last closed field line.

[28] As mentioned before, the thermal catastrophe model predicts a MLT dependence on the solar wind velocity. The region with conditions favorable to the thermal catastrophe is expected to move from premidnight hours toward later times when the solar wind speed increases [Henderson and Murphree, 1995]. The relationship between the 1-hour averaged $\mathrm{V}$ value and the MLT of the onset is presented in Figure 8. It clearly shows an absence of correlation $(\mathrm{R}=$ 0.0003 ) in our dataset. A possible explanation for the correlation suggested by the earlier study is that Viking data were collected over different seasons when both the average solar wind speed and the onset MLT changed independently without any physical link.

\subsection{Interplanetary Magnetic Field}

[29] No correlation was found between onset MLAT and $\mathrm{B}_{\mathrm{x}}(\mathrm{R}=-0.06$, Figure $9 \mathrm{a})$ or $\mathrm{B}_{\mathrm{y}}(\mathrm{R}=0.08$, Figure $9 \mathrm{~b})$. The correlation between the magnetic latitude of onsets and 1-hour averaged IMF $B_{z}$ component is illustrated in Figure 9c. Different symbols are used for onsets following periods with negative (diamonds) or positive (stars) mean $\mathrm{B}_{\mathrm{z}}$ component. For $\mathrm{B}_{\mathrm{z}}>0$, no clear trend is observed, probably owing to the limited sample of cases. For $\mathrm{B}_{\mathrm{z}}<0$, a trend of decreasing MLAT for larger negative values of $B_{z}$ is observed $(R=0.36)$. The relationship

$$
\operatorname{MLAT}=66.39^{\circ}+0.37^{\circ} \times \mathrm{B}_{\mathrm{z}}
$$

indicates that the MLAT of onsets statistically shifts $\sim 0.4^{\circ}$ equatorward for each additional (negative) nT increment. It should be noted that $70 \%$ of the substorm onsets observed during the period of this study occurred for $\mathrm{B}_{\mathrm{z}}<0$, indicating that southward IMF is a more favorable configuration for substorm occurrence. However, a strong northward average IMF component does not preclude the development of substorms, possibly because the IMF turned southward during the 1-hour period of averaging. The large scatter of MLAT values about the regression line suggests that the strength of the $B_{z}$ component is not a key factor in the control of the latitude (and probably distance of the source region in the magnetotail). Some control is expected since breakups are known to occur near the most equatorward arcs of the preonset oval. Observations have shown that the location of the equatorward boundary of the nightside auroral oval shifts equatorward as $\mathrm{B}_{\mathrm{z}}$ increases [Holworth and Meng, 1984]. Therefore although the value of $\mathrm{B}_{\mathrm{z}}$ is important in determining the rate of field line reconnection on the

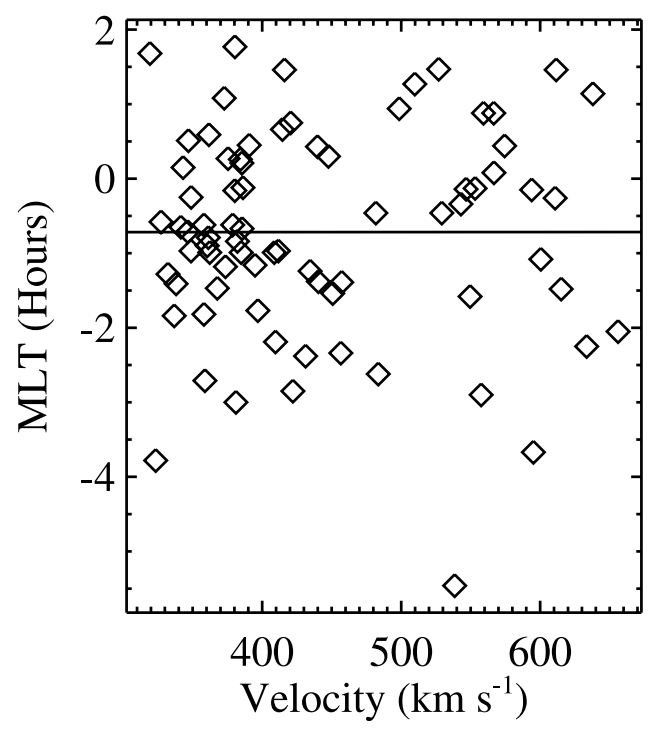

Figure 8. Relationship between the magnetic local time of onsets and the 1-hour averaged solar wind velocity. The horizontal solid line shows the absence of correlation $(\mathrm{R}=$ 0.0003). 

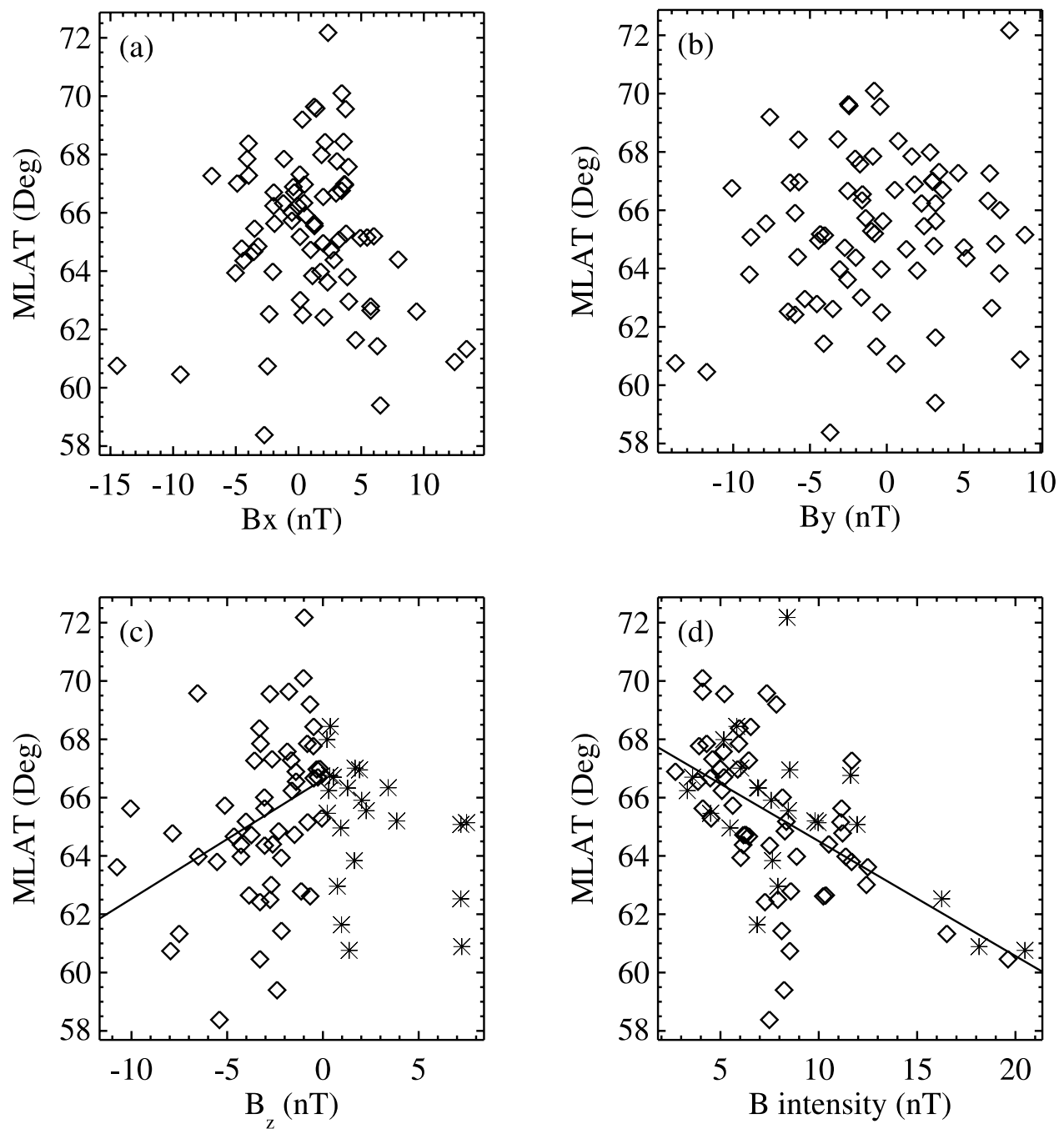

Figure 9. Correlations between the magnetic latitude of onsets and 1-hour averaged interplanetary magnetic field components: $\mathrm{B}_{\mathrm{x}}(\mathrm{a}), \mathrm{B}_{\mathrm{y}}(\mathrm{b}), \mathrm{B}_{\mathrm{z}}(\mathrm{c})$ and $\mathrm{B}$ intensity $(\mathrm{d})$. In Figures $9 \mathrm{c}$ and $9 \mathrm{~d}$, stars and diamonds indicate positive and negative $\mathrm{B}_{\mathrm{z}}$, respectively. The diamonds in Figures $9 \mathrm{c}$ and $9 \mathrm{~d}$ correspond the cases of negative average $B_{z}$ and the stars correspond to positive $B_{z}$.

dayside, it is not clear that it plays a direct role on the location of the substorm initiation.

[30] Figure 9d illustrates the negative correlation between MLAT and the B field intensity values averaged over 1 hour. A relationship with the IMF intensity is observed $(\mathrm{R}=$ $-0.50)$, although a large scatter is observed between 7 and $9 \mathrm{nT}$. The largest B values $(\mathrm{B}>13 \mathrm{nT})$ are clearly associated with MLAT values equal or less than $65^{\circ}$. The linear correlation relationship is given by

$$
\operatorname{MLAT}=68.4^{\circ}-0.39^{\circ} \times \mathrm{B},
$$

where $\mathrm{B}$ is in $\mathrm{nT}$.

[31] The dependence of the magnetic local time of the onset on the IMF was also investigated. The relationship between magnetic local time and $\mathrm{B}_{\mathrm{y}}$ is shown in Figure 10. A statistical shift toward later magnetic local times is observed $(\mathrm{R}=0.33)$ as $\mathrm{B}_{\mathrm{y}}$ (positive eastward) increases and toward early evening for negative $\mathrm{B}_{\mathrm{y}}$, confirming earlier results on that control exerted by the east-west component of the IMF on the local time occurrence of substorms is

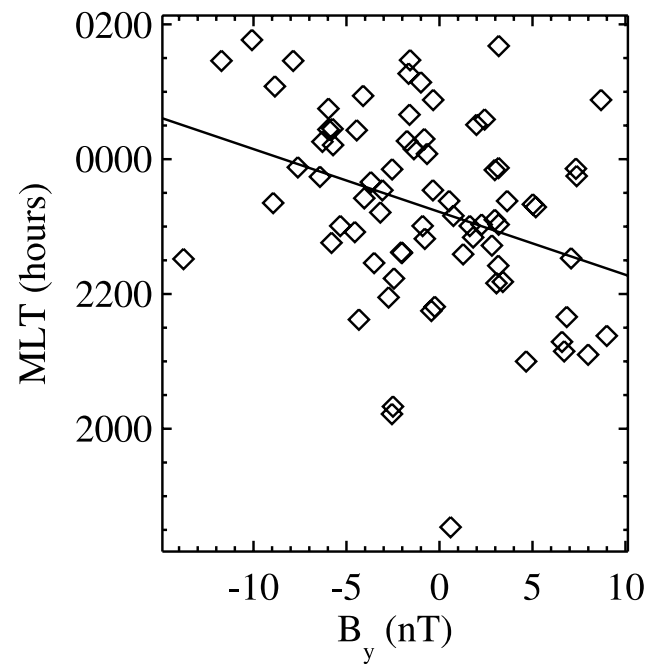

Figure 10. Correlation between the magnetic local time of onsets and the 1-hour averaged interplanetary magnetic $\mathrm{B}_{\mathrm{y}}$ component. 

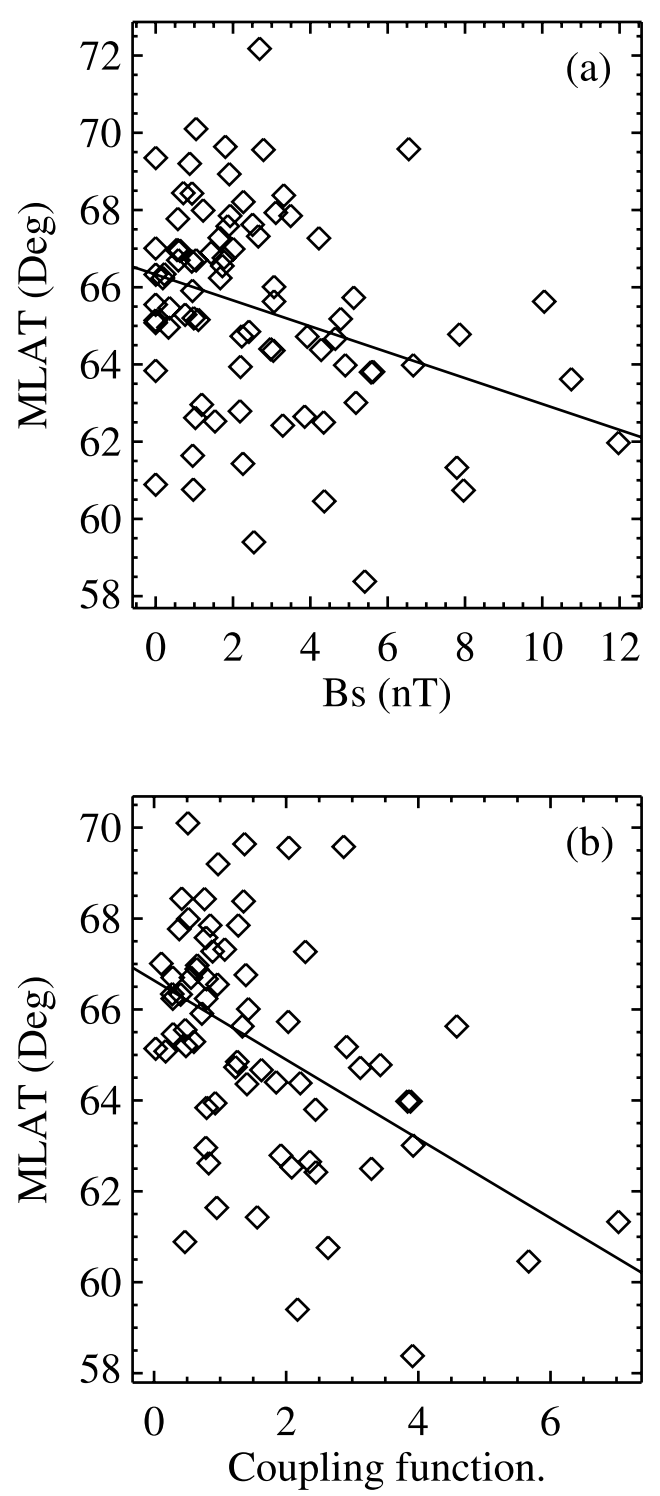

Figure 11. Correlation between the substorm onset latitude and (a) the 1-hour averaged southward IMF $B_{\mathrm{s}}$, (b) the solar wind $\varepsilon$ coupling function (see text).

dominant [Liou et al., 2001]. The linear regression analysis indicates that the rate of MLT eastward displacement is $0.09 \mathrm{hr} / \mathrm{nT}$. As expected, no significant correlation is obtained between MLT and $\mathrm{B}_{\mathrm{x}}$ or $\mathrm{B}_{\mathrm{z}}$.

[32] The importance of the solar wind conditions during the period preceding the onset has been further examined using two indicators of the amount of field line reconnection on the dayside and thus the amount of energy stored in the lobe region. The first one is the time-integrated positive southward IMF component $\mathrm{B}_{\mathrm{S}}$ [Zhou and Tsurutani, 2001]. $B_{s}$ is defined as equal to $\left|B_{z}\right|$ if $B_{z}<0$ and 0 if $B_{z} \geq 0$ and may be used as a proxy for the solar wind input to the magnetosphere. Figure 11a shows the negative correlation $(\mathrm{R}=-0.44)$ between the hourly averaged $\mathrm{B}_{\mathrm{s}}$ value and the onset latitude. The correlation coefficient for $B_{s}$ is higher than for $\mathrm{B}_{\mathrm{z}}$. A second comparison with a more sophisticated coupling function was made using the expression $\varepsilon=\mathrm{P}^{1 / 6} \mathrm{~V}$ $\mathrm{B}_{\mathrm{T}} \sin ^{4}\left(\theta_{\chi} / 2\right)$ described by Vasyliunas et al. [1982] and is illustrated in Figure $11 \mathrm{~b}$. In this expression $\mathrm{B}_{\mathrm{T}}$ is the transverse component of the IMF and $\theta_{\mathrm{c}}$ is the IMF cone angle $=\tan ^{-1}\left(\mathrm{~B}_{\mathrm{T}} / \mathrm{B}_{\mathrm{x}}\right)$. It is based on the assumption that the energy storage in the magnetosphere is proportional to the solar wind kinetic energy intercepted by an energy collection region on the magnetopause. This expression was shown by Liou et al. [1998] to provide the highest level of correlation between solar wind parameters and the total amount of auroral precipitation on the nightside. Figure $11 \mathrm{~b}$ shows that the correlation $(\mathrm{R}=-0.47)$ is slightly better than with $B_{s}$ but is not higher than with the B field intensity.

\section{Discussion and Summary}

[33] The distribution of the magnetic latitude and local times of the substorm onsets observed during the winter solstice period confirms earlier finding obtained with FUV imagers. The median MLAT values are $\sim 1.4^{\circ}$ lower than these found by Liou et al. [2001] for POLAR observations at winter periods. They are intermediate between the DE-I value obtained by Craven and Frank [1991] and the Viking observations by Henderson and Murphree [1995]. The origin of these differences is unclear. They may be partly ascribed to the uncertainties of the satellite pointing, different averaged solar wind conditions and true seasonal effects. For example, the data analyzed in this study were obtained very close to winter solstice, in contrast to the seasonal averaging of the DE-I or Viking studies. The winter POLAR data were collected during the full year and showed intrinsic seasonal variations of the onset latitude presumably as a consequence of changes between the Earth's dipole angle and the IMF. Our median magnetic local time is later than in previous studies. This result is however compatible with the conclusions of the seasonal study of Liou et al. [2001] who found that the median MLT shifts by approximately 1 hour from summer to winter solstice. This shift in MLT is probably linked to a seasonal variation of the IMF $B_{y}$ component which tends to be more positive and thus to shift onsets toward earlier later times than at other seasons. The period of our observations close to winter solstice probably explains the larger concentration near magnetic midnight. The asymmetry of the MLAT distribution about the peak and the more symmetric MLT distribution is also in line with the results from POLAR showing a more extended tail in the global (all seasons) distribution of onset MLAT.

[34] Neither MLAT nor MLT distributions of electron and proton onsets were found significantly different, considering uncertainties. This result indicates that at the spatial scale of the observations $(\sim 100 \mathrm{~km})$, electrons and protons causing the initial brightening originate from the same region of the magnetotail. The observations reported here are based on a perspective that is different from groundbased meridian scanning photometers or all-sky cameras restricted to a given location in the oval and not necessarily colocated with the initial breakup. The IMAGE-FUV observations provide simultaneous snapshots of the electron and proton dynamics that allow untangling the space-time ambiguity of ground-based measurements.

[35] The effect of the IMF $B_{z}$ on the onset latitude is in partial agreement with the conclusions of Liou et al. [2001]. We find that the latitude of the onsets decreases with increasingly negative $B_{z}$. For positive $B_{z}$, our results are 
not so clear, probably as a consequence of the limited sample size. The highest correlation with the IMF characteristics was found with the B field intensity. Negative correlations are also observed with two proxies of the amount of IMF reconnection on the dayside. However, the highest correlation was found with the total IMF B field intensity.

[36] The relationship between the onset latitude and the solar wind velocity, density, and dynamic pressure had apparently not been investigated before. The IMF magnitude and orientation controls the rate of reconnection on the dayside of the magnetosphere whereas $\mathrm{P}_{\mathrm{dyn}}$ determines the rate of compression. One can speculate that the dynamic pressure modifies the shape of the magnetosphere and therefore the mapping of a given region of the magnetosphere into the ionosphere. Other studies using magnetic field mapping indicate that most onsets occur within $10 R_{E}$ and their distance is inversely related to the solar wind activity and IMF strength. This result is also consistent with earlier observations [Mauk and McIlwain, 1974; Lopez et al., 1989] showing that the substorm injection boundary of low energy electrons shifts earthward as Kp increases. The IMF direction determines the dayside reconnection rate that seems to have less effect on the location of substorm onsets.

[37] Three elements from this study that constrain the locations of the instability processes leading to triggering of auroral onsets may be summarized as follows: (1) all observed breakups take place inside the region of proton precipitation observed with the SI12 imager during the growth phase, (2) the distance between the latitude of onsets (both for electrons and protons) and the poleward boundary of the FUV auroral emission increases when onsets occur at lower MLAT, (3) breakups occur poleward of and at a nearly fixed distance from the equatorward boundary of the FUV oval.

[38] One conclusion is that the latitudinal motion of the onsets with increasing $\mathrm{B}$ or $\mathrm{P}_{\mathrm{dyn}}$ is not simply a consequence of the change of the polar cap size during the period preceding the onset. The equatorward displacement of the onset does not follow the change of latitudinal position of the polar cap boundary controlled by solar wind activity, mostly $B_{Z}$. Instead, it tends to follow the variations of the distance of the Earthward boundary of the main plasma sheet. The nearly constant distance between the onsets and the equatorward boundary of the oval suggests that as the oval expands equatorward with increasing amount of energy transferred to the magnetosphere, the onsets also occur at lower latitudes.

[39] Our observations suggest that the mechanism leading to the breakup vortex occurs in a region located on stretched field lines as confirmed by the relative position of the onset and the equatorward boundary of the region of proton precipitation. In all cases examined for this study and other substorms observed at other periods, the onset is immerged into a preexisting region of SI12 Ly- $\alpha$ emission due to energetic proton precipitation. As previously pointed out on the basis of ground based measurements by Samson et al. [1992], the auroral onset location inside regions of proton precipitation is a strong evidence that breakups take place on stretched closed field lines, close to the Earth $\left(<10 \mathrm{R}_{\mathrm{E}}\right)$. These results are consistent with a preliminary study of Geotail proton data by Voronkov et al. [2002] indicating that the average position of the tailward boundary of the hot
Table 1. Median Distribution of Electron Onsets Observed With Global FUV Imagers

\begin{tabular}{llcc}
\hline \multicolumn{1}{c}{ Authors } & \multicolumn{1}{c}{ Imager } & MLAT & MLT \\
\hline Craven and Frank [1991] & DE-I & $65^{\circ}$ & 2250 \\
Henderson and Murphree [1995] & Viking UV imager & $66.7^{\circ}$ & 2305 \\
Liou et al. [2001] & UVI-Polar (winter) & $67^{\circ}$ & 2300 \\
This work & FUV-IMAGE (winter) & $65.6^{\circ}$ & 2324 \\
\hline
\end{tabular}

proton population is close to $10 \mathrm{R}_{\mathrm{E}}$. The interpretation of substorm onset observations by Murphree et al. [1993] led to similar conclusions. They found that the average GSM $\times$ value of the mapped onset were $\sim 7.8 \mathrm{R}_{\mathrm{E}}$. They also noted that the onsets are located well equatorward of the boundary between open and closed field lines. Frank and Sigwarth [2000] found that the onsets mapped to the ring current region between 5 and $9 \mathrm{R}_{\mathrm{E}}$. They independently verified that the brightening of one of the events occurred inside the region of proton precipitation from the ring current as determined from the hydrogen $\mathrm{H} \alpha$ auroral emission. Based on a combination of in situ and comprehensive ground data, Dubyagin et al. [2003] concluded that on isolated substorm onset was initiated near $8 \mathrm{R}_{\mathrm{E}}$, just $0.4^{\circ}$ poleward of the proton IB. Our observations that the onsets are always imbedded within precipitation of ring current protons strongly confirms those earlier findings (Table 1).

[40] Acknowledgments. The authors thank a reviewer for useful suggestions. JCG acknowledges support from the Belgian Fund for Scientific Research (FNRS). The Fund for Collective Fundamental Research (FRFC) and the PRODEX program of ESA provided financial support for this research to the University of Liège. The work at UC Berkeley was supported through SWRI subcontract 83820 at the University of California, Berkeley and by NASA under contract NAS5-96020. ACE level 2 data were provided by N. F. Ness (MFI) and D. J. McComas (SWEPAM) through the ACE Science Center and CDAweb.

[41] Arthur Richmond thanks the reviewers for their assistance in evaluating this paper.

\section{References}

Akasofu, S. I. (1964), The development of the auroral substorms, Planet. Space Sci., 12, 273.

Baker, J. B., C. R. Clauer, A. J. Ridley, V. O. Papitashvili, M. J. Brittnacher, and P. T. Newell (2000), The nightside poleward boundary of the auroral oval as seen by the MSP and the Ultraviolet Imager, J. Geophys. Res., $105,21,267$.

Baker, D. N., et al. (2002), Timing of magnetic reconnection initiation during a global magnetospheric substorm onset, Geophys. Res. Lett., 29(24), 2190, doi:10.1029/2002GL015539.

Brittnacher, M. J., M. Fillingim, G. Parks, G. Germany, and J. Spann (1999), Polar cap area and boundary motion during substorms, J. Geophys. Res., 104, 12,251.

Burch, J. L., et al. (2001), Views of Earth's magnetosphere with the IMAGE satellite, Science, 291, 619.

Craven, J. D., and L. A. Frank (1991), Diagnosis of auroral dynamics using global auroral imaging with emphasis on large-scale evolutions, in Auroral Physics, edited by C.-I. Meng, M. J. Rycroft, and L. A. Frank, p. 273, Cambridge Univ. Press, New York.

Donovan, E. F., B. J. Jackel, I. Voronkov, T. Sotirelis, F. Creutzberg, and N. A. Nicholson (2003), Ground-based optical determination of the b2i boundary: A basis for an optical MT-index, J. Geophys. Res., 108(A3), 1115, doi:10.1029/2001JA009198

Dubyagin, S. V., V. A. Sergeev, C. W. Carlson, S. R. Marple, T. I. Pulkkinen, and A. G. Yahnin (2003), Evidence of near-Earth breakup location, Geophys. Res. Lett., 30(6), 1282, doi:10.1029/2002GL016569.

Frank, L. A., and J. D. Craven (1988), Imaging results from Dynamics Explorer I, Rev. Geophys., 26, 249.

Frank, L. A., and J. B. Sigwarth (2000), Findings concerning the positions of substorm onsets with auroral images from the Polar spacecraft, J. Geophys. Res., 105, 12,747.

Frey, H. U., T. J. Immel, S. B. Mende, J.-C. Gérard, B. Hubert, S. Habraken, J. Spann, and G. R. Gladstone (2003), Summary of quantitative inter- 
pretation of IMAGE far ultraviolet auroral data, Space Sci. Rev., 109 , 255.

Galperin, Y. I., and Y. I. Feldstein (1991), Auroral luminosity and its relationship to magnetospheric plasma domains, in Auroral Physics, edited by C.-I. Meng, M. J. Rycroft, and L. A. Frank, p. 207, Cambridge Univ. Press, New York

Gérard, J. C., B. Hubert, D. V. Bisikalo, V. I. Shematovich, H. U. Frey, S. B. Mende, G. R. Gladstone, and C. W. Carlson (2001), Observation of the proton aurora with IMAGE-FUV and simultaneous ion flux in situ measurements, J. Geophys. Res., 106, 28,939.

Gladstone, R. G., et al. (2000), Stellar calibration of the WIC and SI imagers and the GEO photometer on IMAGE/FUV, Eos Trans. $A G U$, 81(48), Fall Meet. Suppl., Abstract SM72A-06.

Goertz, C. K., and R. A. Smith (1989), The thermal catastrophe model of substorms, J. Geophys. Res., 94, 6581.

Henderson, M. G., and J. S. Murphree (1995), Comparison of Viking onset locations with the predictions of the thermal catastrophe model, J. Geophys. Res., 100, 21,857.

Holworth, R. H., and C.-I. Meng (1984), Auroral boundary variations and the interplanetary magnetic field, Planet. Space. Sci., 32, 25.

Hubert, B., J. C. Gérard, D. S. Evans, M. Meurant, S. B. Mende, H. U. Frey, and T. J. Immel (2002), Total electron and proton energy input during auroral substorm: Remote sensing with IMAGE-FUV, J. Geophys. Res., 107(A8), 1183, doi:10.1029/2001JA009229.

Kauristie, K., J. Weygand, T. I. Pulkkinen, J. S. Murphree, and P. T. Newell (1999), Size of the auroral oval: UV ovals and precipitation boundaries compared, J. Geophys. Res., 104, 2321.

Liou, K., P. T. Newell, C.-I. Meng, M. Brittnacher, and G. Parks (1998), Characteristics of the solar wind controlled auroral emissions, J. Geophys. Res., 103, 17,543.

Liou, K., C.-I. Meng, T. Y. Lui, P. T. Newell, M. Brittnacher, G. Parks, G. D. Reeves, R. R. Anderson, and K. Yumoto (1999), On relative timing in substorm onset signatures, J. Geophys. Res., 104, $22,807$.

Liou, K., P. T. Newell, D. G. Sibeck, C. I. Meng, M. Brittnacher, and G. Parks (2001), Observation of IMF and seasonal effects in the location of auroral substorms, J. Geophys. Res., 106, 5799.

Lopez, R. E., A. T. Y. Lui, D. G. Sibeck, K. Takahsahi, R. W. McIntire, L. J. Zanetti, and S. M. Krimigis (1989), On the relationship between the energetic particle flux morphology and the change in the magnetic field magnitude during substorms, J. Geophys. Res., 94, 17,105.

Lui, A. T. Y. (2001), Current controversies in magnetospheric physics, Rev. Geophys., 39, 535.

Lyons, L. R., I. O. Voronkov, E. F. Donovan, and E. Zesta (2002), Relation of substorm breakup arc to other growth-phase auroral arcs, J. Geophys. Res., 107(A11), 1390, doi:10.1029/2002JA009317.
McPherron, R. L., C. T. Russell, and M. P. Aubry (1973), Satellite studies of magnetospheric substorms on August 15, 1968: 9. Phenomenological model for substorms, J. Geophys. Res., 78, 3131.

Mauk, B. H., and C. E. McIlwain (1974), Correlation of Kp with the substorm-injected plasma, J. Geophys. Res., 79, 3193.

Mende, S. B., et al. (2000), Far ultraviolet imaging from the IMAGE spacecraft: 1. System design, Space Sci. Rev, 91, 243.

Mende, S. B., C. W. Carlson, H. U. Frey, L. M. Peticolas, and N. Østgaard (2003), FAST and IMAGE-FUV observations of a substorm onset, J. Geophys. Res, 108(A9), 1344, doi:10.1029/2002JA009787.

Meng, C. I., B. Tsurutani, K. Kawasaki, and S. I. Akasofu (1973), Crosscorrelation analysis of the AE index and the interplanetary magnetic field $\mathrm{B}_{\mathrm{z}}$ component, J. Geophys. Res., 78, 617.

Murphree, J. S., R. D. Elphinstone, M. G. Henderson, L. L. Cogger, and D. J. Hearn (1993), Interpretation of optical substorm onset observations, J. Atmos. Terr. Phys., 55, 1159.

Newell, P. T., Y. I. Feldstein, Y. I. Galperin, and C. I. Meng (1996), Morphology of nightside precipitation, J. Geophys. Res., 101, 10,737.

Richmond, A. D. (1995), Ionospheric electromagnetics using magnetic apex co-ordinates, J. Geomagn. Geoelectr., 47, 191.

Samson, J. C., L. Lyons, P. T. Newell, and F. Creutzberg (1992), Proton aurora and substorm intensifications, Geophys. Res. Lett., 19, 2167.

Shiokawa, K., W. Baumjohann, and G. Haeredel (1997), Breaking of high speed flows in the near-Earth tail, Geophys. Res. Lett., 24, 1179.

Tsyganenko, N. A. (1987), Global quantitative model of the geomagnetic field in the cislunar magnetosphere for different disturbance levels, Planet. Space Sci., 95, 1347.

Vasyliunas, V. M., J. R. Kan, G. L. Siscoe, and S. I. Akasofu (1982), Scaling relations governing magnetospheric energy transfer, Planet. Space Sci., 30, 359.

Voronkov, I. O., E. F. Donovan, P. Dobias, J. C. Samson, and L. R. Lyons (2002), Near-Earth breakup in substorms: Empirical and model constraints, in Proceedings of the Sixth International Conference on Substorms, edited by R. M. Winglee, p. 270, Univ. of Wash., Seattle, Wash.

Voronkov, I. O., E. F. Donovan, and J. C. Samson (2003), Observations of the phases of the substorm, J. Geophys. Res., 108(A2), 1073, doi:10.1029/2002JA009314.

J.-C. Gérard, A. Grard, B. Hubert, and M. Meurant, Laboratoire de Physique Atmosphérique et Planétaire, Université de Liège, Allèe du 6 Août, 17 B-4000 Liège, Belgium. (jc.gerard@ulg.ac.be; a.grard@ulg.ac.be; b.hubert@ulg.ac.be; mmeurant@ulg.ac.be)

S. B. Mende, Space Science Laboratory, University of California, Berkeley, Centennial Drive at Grizzly Peak Blvd., Berkeley, CA 947207450, USA. (mende@ssl.berkeley.edu) 